\title{
Optical-feedback-induced stability and instability in broad-area semiconductor lasers
}

\author{
Yasuhiro Fujita and Junji Ohtsubo ${ }^{\text {a) }}$ \\ Faculty of Engineering, Shizuoka University, 3-5-1 Johoku, Hamamatsu 432-8561. Japan
}

(Received 22 March 2005; accepted 6 June 2005; published online 15 July 2005)

\begin{abstract}
Stable and unstable oscillations of broad-area semiconductor laser with optical feedback are experimentally examined. We observe stable oscillations and also coexistent states between stable oscillations and low-frequency fluctuations depending on the feedback positions of the laser beam in the active layer. The laser shows single mode operations both for the oscillation frequency and the spatial mode when it is stabilized. (c) 2005 American Institute of Physics. [DOI: 10.1063/1.1999850]
\end{abstract}

High power semiconductor lasers are the promising devices as light sources for second-harmonic generations, laser cutting, and laser fusions due to compactness, high conversion efficiency from electric to optical power, and inexpensive device. ${ }^{1}$ To attain high power laser as a single element, broad-area semiconductor laser which has a broad stripe width has been developed. Ordinary edge-emitting semiconductor lasers used for optical communications and optical data storage systems have narrow stripe width of less than several micron meters, while the stripe width of broad-area semiconductor laser is ten times or more larger than those of ordinary edge-emitting semiconductor lasers. Therefore, broad-area semiconductor laser can emit great optical power as much as $10 \mathrm{~W}$ or more in a single source.

In spite of the attractive features for applications, the beam quality of broad-area semiconductor lasers is rather poor compared with other high power lasers, such as glass lasers and $\mathrm{CO}_{2}$ lasers. ${ }^{2-7}$ Due to the addition of the extra degree of freedom to ordinary edge-emitting semiconductor lasers along the stripe width, broad-area semiconductor laser is no more stable laser and shows instabilities in its optical output even in the solitary oscillation. For example, a fast fluctuation known as filamentation is a typical instability of broad-area semiconductor laser even observed in its solitary oscillation. ${ }^{4}$ The instabilities are much enhanced by the introduction of extra degree of freedoms such as optical feedback, optical injection, and modulation. ${ }^{8}$ Low-frequency fluctuation (LFF) is one of unstable intermittent oscillations observed in a broad-area semiconductor laser subjected to optical feedback. ${ }^{7}$ In the meantime, the method of optical feedback is frequently used to stabilize unwanted fluctuations in broad-area semiconductor lasers. ${ }^{9-15}$

In the previous experimental report, LFFs were observed in a broad-area semiconductor laser subjected both for conventional and phase-conjugate optical feedback. ${ }^{7}$ Thus, LFFs are not special features observed in edge-emitting narrowstripe semiconductor lasers under limited circumstances, but they are widely observed in semiconductor lasers with various device structures including broad-area semiconductor lasers. In this letter, we experimentally observed LFFs as unstable characteristics of broad-area semiconductor lasers with optical feedback. Also stable oscillations and coexistent states between stable and LFF oscillations were observed in the laser output power. Both the optical frequency and the

${ }^{a)}$ Electronic mail: tajohts@ipc.shizuoka.ac.jp spatial mode were stabilized to single modes at the stable oscillations.

In the experiments, we used a SONY SLD301V gainguided broad-area semiconductor laser of AlGaAs quantumwell structure with oscillation wavelength of $780 \mathrm{~nm}$ and a maximum output power of $100 \mathrm{~mW}$. The width of the active region was $50 \mu \mathrm{m}$ and the threshold injection current was $143 \mathrm{~mA}$. At the solitary state, the laser oscillated at multimodes both for the optical frequency and the spatial modes. The bias injection current of the laser was fixed by a stabilized current source driver and the temperature of the laser was also controlled by an automatic temperature control circuit. The emitted light from the laser was collimated by a set of cylindrical lenses (focal lengths of $f=3.9$ and $f=50 \mathrm{~mm}$ ) and a part of the beam was fed back from an external reflector into the active layer. The feedback strength could be adjusted by a neutral density filter inserted in the optical path. The external mirror was positioned at $30 \mathrm{~cm}$ away from the front facet of the laser. The external feedback fraction in front of the collimated lens of the laser beam was $5 \%$ of the original oscillation intensity but the actual amount of the feedback to the active layer was much less than this fraction, since there were losses of light due to the coupling between the internal and external fields and the diffractions by the optical components. By the optical feedback, the threshold of the injection current was reduced as much as $13.8 \%$ from the solitary level.

The other beam divided by the beam splitter was fed into diagnostic systems. The beam was detected by a high-speed photodetector (NEW FOCUS 1537M-LF: bandwidth of 6.5 $\mathrm{GHz}$ ) and the photocurrent from the detector was fed into a fast digital oscilloscope (HP 54845A: bandwidth of $1.5 \mathrm{GHz}$ ) to analyze chaotic time series. Also, microwave frequency characteristics were examined by a rf spectrum analyzer (HP 8595E: bandwidth of $6.5 \mathrm{GHz}$ ) to analyze their Fourier components. The beam was also analyzed by an optical spectrum analyzer (ADVANTEST Q8344A, maximum resolution of $0.05 \mathrm{~nm}$ ) to check their optical oscillation frequency. The far-field patterns of the spatial modes were also captured by a CCD camera.

We found completely stable laser oscillations of the broad-area laser with strong optical feedback at low bias injection current close to but below the laser threshold (the injection current of $140 \mathrm{~mA}$ ). Figure $1(\mathrm{a})$ is the time series of the output from the broad-area semiconductor laser at the solitary oscillation. Since the laser was biased below the 


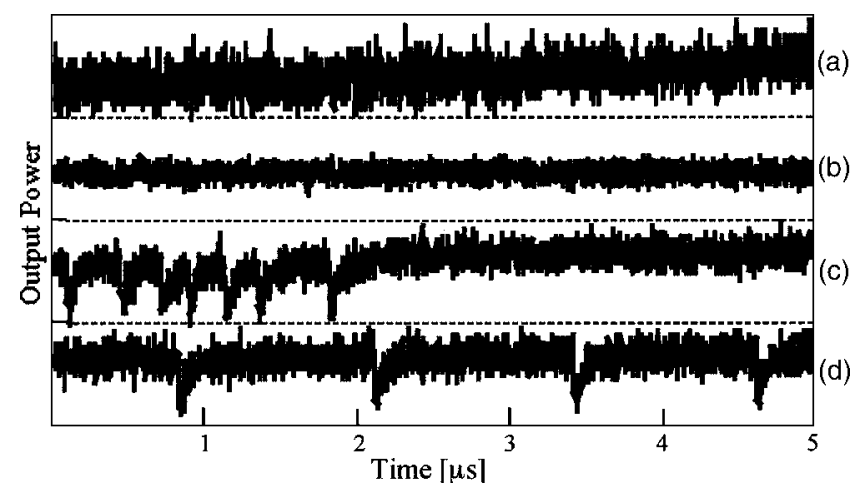

FIG. 1. Laser output powers by the tilt of the external mirror: (a) solitary oscillation. (b) stable oscillation; (c) coexistent state; and (d) fully developed LFFs in the presence of optical feedback.

threshold, much noises originated from spontaneous emissions were induced in the output power. With the introduction of optical feedback at this bias injection current, we obtained completely constant output power as shown in Fig. 1(b). As easily understood from the figure, a lasing oscillation started and the noise level was reduced compared with that of Fig. 1(a). The oscillation was completely stable and it was different from a transient between stable and coexistent states, which is discussed in the following. At this stable state, each spatial mode was supposed to be fed back into the corresponding position in the active layer. Under this operating condition, the laser showed single-mode operations both for the oscillation frequency and the spatial mode. The stabilization of semiconductor lasers induced by optical feedback has been reported not only in narrow-stripe semiconductor lasers but also in broad-area semiconductor lasers and semiconductor laser arrays. ${ }^{12,14}$ Also selective optical feedback for the spatial modes has been frequently used for stabilization of broad-area semiconductor lasers. ${ }^{14}$ The mechanism of the stabilization can be considered as the same as that for narrow-stripe semiconductor lasers, although broadarea semiconductor laser is essentially a multimode laser.

By the introduction of a tilt of the external reflector along the active layer, we observed coexistent states between stable states and LFF oscillations. The beam shift induced by the tilt was roughly estimated to be several to $10 \mu \mathrm{m}$ from the center of the active region. The conditions of the bias injection current and the external feedback were the same as before except for the tilt. Figure 1(c) shows an example of the laser output for coexistent states. The durations between stable and unstable oscillations were irregular in time and it was on the order of milliseconds. In a narrow-stripe semiconductor laser, LFFs are the typical instability induced by optical feedback and they are frequently observed at a low bias injection current, even for either single-mode or multimode oscillations. In the meantime, there are coexistent chaotic states of different attractors in which the system behaves differently in accordance with the initial different starting conditions, in spite of the same operating parameters of the nonlinear system. Indeed, coexistent states between stable states and LFF oscillations have been experimentally observed in a narrow-stripe semiconductor laser with a singlemode operation. ${ }^{16,17}$ However, little has been studied for the dynamics for broad-area semiconductor lasers and this is an observation of coexistent states in broad-area semiconductor

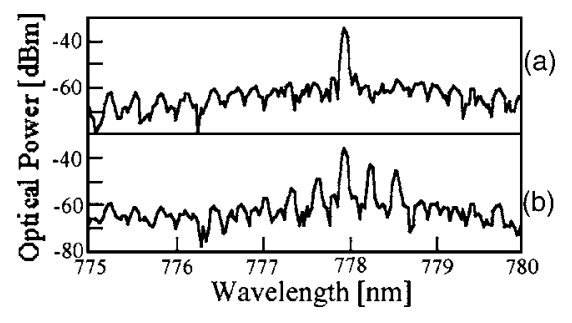

FIG. 2. Optical spectra of laser oscillations: (a) and (b) correspond to Figs. $1(\mathrm{~b})$ and $1(\mathrm{~d})$, respectively.

laser which essentially oscillates at multimodes both for the oscillation frequency and the spatial modes.

Heil et al. experimentally obtained a map of stable oscillations, unstable LFFs, coherence collapse states, and coexistent states in the phase space of the injection current and the feedback strength in a narrow-stripe semiconductor laser with optical feedback. ${ }^{16}$ They also pointed out that the occurrences of coexistent states were strongly dependent on the $\alpha$-parameter which is an important parameter of semiconductor lasers. ${ }^{17}$ According to their studies, coexistent states were typically observed at low bias injection current with strong optical feedback. With the decrease of the feedback strength, the laser switched to LFF oscillations from coexistent states, and it reached to coherence collapse states for the further decrease of the strength. It was reported that coexistent states of chaotic oscillations were also observed in narrow-stripe semiconductor lasers with multimode oscillations. ${ }^{16}$ In addition to the characteristics of narrow-stripe semiconductor lasers, broad-area semiconductor lasers have different features originated from the existence of the broad active area. Therefore, the laser oscillations in broad-area semiconductor lasers are much affected by the spatial and temporal instabilities such as filamentation induced by the hole-burning effect of carriers in the active region. Also there is a coupling among spatial modes induced by optical feedback. However, the mechanism for the occurrence of coexistent states in broadarea semiconductor lasers is considered to be almost the same as that in narrow-stripe semiconductor lasers. As usual, a particular state of different chaotic attractors can be only accessible in a real experiment for the corresponding starting point among the different attractors. But the existence of the extra conditions for the oscillations in a broad-area semiconductor laser makes it easier to switch one state to the other. But the further study is still required to fully understand the characteristics and the origin of coexistent states in broadarea semiconductor lasers.

For the further increase of the tilt angle (the shift of the beam in the active region was around $15 \mu \mathrm{m}$ from the center), stable transients disappeared and only LFF oscillations were observed as shown in Fig. 1(d). Due to the increase of the tilt angle, the effective feedback strength was reduced compared with the previous case. The frequency of LFFs was smaller than that in the coexistent state in Fig. 1(c), since the feedback strength decreased. In the previous experimental results, the laser oscillation evolved into LFF oscillations from coexistent states by the reduction of the optical feedback strength. ${ }^{16}$ However, we could only observed the transition to LFF oscillations from coexistent states only by a tilt of the external mirror in the broad-area semiconductor lasers.

Figure 2 shows the optical spectra observed by the optical spectrum analyzer corresponding to the time series for AIP license or copyright; see http://apl.aip.org/apl/copyright.jsp 


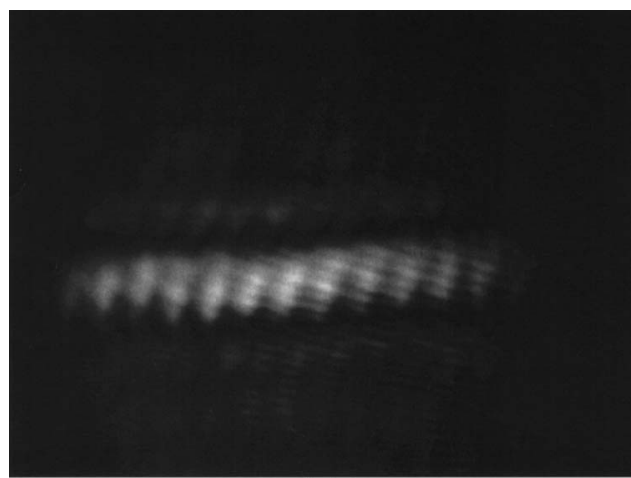

(a)

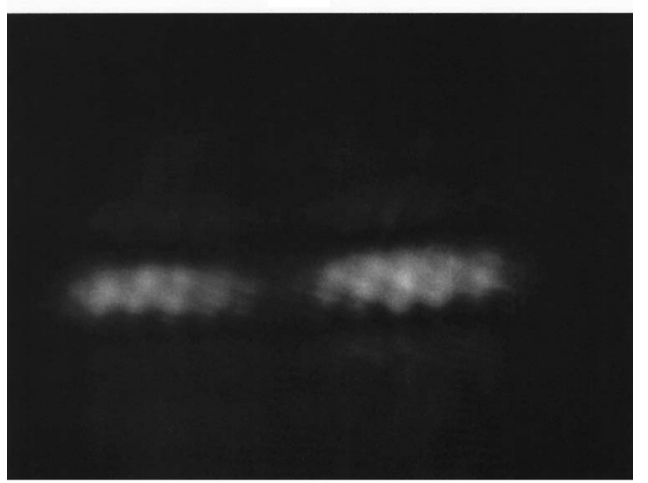

(b)

FIG. 3. Spatial mode patterns: (a) stable and (b) fully developed LFF states corresponding to Figs. 1(b) and 1(d), respectively.

the stable and unstable oscillations in Fig. 1. A single oscillation peak was detected for the stable oscillation as shown in Fig. 2(a) [corresponding to the time series in Fig. 1(b)]. In this case, the suppression ratio of side modes was greater than $20 \mathrm{~dB}$ and the laser oscillated almost at a single mode. But, at the coexistent state in Fig. 1(c), the averaged spectrum over time was a multimode due to the inclusion of LFF oscillations (not shown here). The laser also showed multimode oscillation in the complete LFF regime as shown in Fig. 2(b) [corresponding to the time series in Fig. 1(d)], but the oscillation lines were different from those of the coexistent state. Figures 3(a) and 3(b) show the far-field patterns of the spatial modes (the actual size of the beam was $7 \mathrm{~mm}$ long, which was produced from the collimation by a set of the cylindrical lenses) for the stable and coexistent states corresponding to Figs. 1(b) and 1(d), respectively. The farfield pattern had almost a single-peak for the stabilized case as shown in Fig. 3(a). Thus the laser was stabilized to single modes both for the transverse and longitudinal modes in the presence of optical feedback. At the LFF oscillations, the laser had a twin-peaked far-field pattern [Fig. 3(b)]. A twinpeaked far-field pattern is a typical feature of the laser beam profile for a solitary operation in a broad-area semiconductor lasers at low bias injection current. We can see fringe pattern in the figure, but this is induced by the interference of the cover glass in front of the CCD device in the camera and they are not related to laser dynamics.

We have examined stable and unstable behaviors in broad-area semiconductor lasers with optical feedback. The stable and unstable LFF oscillations were observed at close but less than the laser threshold this time. However, LFFs are the characteristics in broad-area semiconductor lasers observed not only at low bias injection current but also higher bias injection current. ${ }^{7}$ LFF is one of instabilities on the route to intermittent chaos, therefore we may expect stabilization of lasers on such a route even at higher bias injection current in the practical use. A little has been studied for the dynamics of LFFs and coexisting states in broad-area semiconductor lasers. The detailed studies are still expected for understanding the dynamics and stabilizing broad-area semiconductor lasers.

The authors would like to thank Y. Takiguchi for their fruitful discussions.

${ }^{1}$ R. Diehl, High-Power Diode Laser, Fundamentals, Technology, Applications (Springer, Berlin, 2000).

${ }^{2}$ O. Hess, S. W. Koch, and J. V. Moloney, IEEE J. Quantum Electron. 31, 35 (1995).

${ }^{3}$ I. Fischer, O. Hess, W. Elsässer, and E. Göbel, Europhys. Lett. 35, 579 (1996).

${ }^{4}$ J. R. Marciante and G. P. Agrawal, IEEE J. Quantum Electron. 32, 590 (1996).

${ }^{5}$ O. Hess and T. Kuhn, Phys. Rev. A 54, 3360 (1996).

${ }^{6}$ T. Burkhard, M. O. Ziegler, I. Fischer, and W. Elsässer, Chaos, Solitons Fractals 10, 845 (1999).

${ }^{7}$ J. S. Lawrence and D. M. Kane, J. Lightwave Technol. 20, 100 (2002).

${ }^{8}$ J. Ohtsubo, Progress in Optics, edited by E. Wolf (North-Holland, Amsterdam, 2002), Vol. 44, Chap. 1.

${ }^{9}$ Y. Champagne, S. Mailhot, and N. McCarth, IEEE J. Quantum Electron. 31, 795 (1995).

${ }^{10}$ J. Martín-Regalado, G. H. M. van Tartwijk, S. Balle, and M. San Miguel, Phys. Rev. A 54, 5386 (1996).

${ }^{11}$ C. Simmendinger, M. Uünkel, and O. Hess, Chaos, Solitons Fractals 10, 851 (1999).

${ }^{12}$ C. Simmendinger, D. Preier, and O. Hess, Opt. Express 5, 48 (1999).

${ }^{13}$ S. Mailhot, Y. Champagne, and N. McCarthy, Appl. Opt. 39, 6806 (2000).

${ }^{14}$ S. Wolff and H. Fouckhardt, Opt. Express 7, 222 (2000).

${ }^{15}$ S. Wolff, A. Rodionov, V. E. Sherstobitov, and H. Fouckhardt, IEEE J. Quantum Electron. 39, 448 (2003).

${ }^{16}$ T. Heil, I. Fischer, and W. Elsässer, Phys. Rev. A 58, R2672 (1998).

${ }^{17}$ T. Heil, I. Fischer, and W. Elsässer, Phys. Rev. A 60, 634 (1999). 